\title{
Correction to: Principles of Exercise Prescription, and How They Influence Exercise-Induced Changes of Transcription Factors and Other Regulators of Mitochondrial Biogenesis
}

\author{
Cesare Granata $^{1,2}$ (1) $\cdot$ Nicholas A. Jamnick $^{1}$ (D) David J. Bishop ${ }^{1,3}$ ()
}

Published online: 7 May 2018

(C) Springer International Publishing AG, part of Springer Nature 2018

\section{Correction to: Sports Med}

https://doi.org/10.1007/s40279-018-0894-4

Section 3, paragraph 3, sentence 1: The following sentence, which previously read:

"The recommended test to determine $\dot{W}_{\max }$ (and the maximum rate of oxygen consumption $\dot{\mathrm{V}}_{2 \max }$ ) is a short (8-12 min) incremental exercise test (IET), where exercise intensity is increased continuously (e.g., 1 week every $2 \mathrm{~s}$ ) or stepwise (e.g., 30 week every $1 \mathrm{~min}$ ) [55] (Fig. 1)."

Should read:

“The recommended test to determine $\dot{W}_{\max }$ (and the maximum rate of oxygen consumption $\dot{\mathrm{V}}_{2 \max }$ ) is a short (8-12 min) incremental exercise test (IET), where exercise intensity is increased continuously (e.g., $1 \mathrm{~W}$ every $2 \mathrm{~s}$ ) or stepwise (e.g., $30 \mathrm{~W}$ every $1 \mathrm{~min}$ ) [55] (Fig. 1)."

The original article has been updated.

The original article can be found online at https://doi.org/10.1007/ s40279-018-0894-4.

Cesare Granata

cesare.granata@monash.edu

1 Institute of Sport, Exercise and Active Living (ISEAL),

College of Sport and Exercise Science, Victoria University,

Melbourne, Australia

2 Department of Diabetes, Central Clinical School, Faculty of Medicine, Nursing and Health Sciences, Monash University, Alfred Centre, 99 Commercial Rd, Melbourne, VIC 3004. Australia

3 School of Medical and Health Sciences, Edith Cowan University, Joondalup, Australia 